\title{
Positive Solutions of a Two-Point Boundary Value Problem for Singular Fractional Differential Equations in Banach Space
}

\author{
Bo Liu and Yansheng Liu \\ Department of Mathematics, Shandong Normal University, Jinan 250014, China \\ Correspondence should be addressed to Yansheng Liu; yanshliu@gmail.com
}

Received 29 May 2013; Accepted 11 July 2013

Academic Editor: William P. Ziemer

Copyright ( 2013 B. Liu and Y. Liu. This is an open access article distributed under the Creative Commons Attribution License, which permits unrestricted use, distribution, and reproduction in any medium, provided the original work is properly cited.

\begin{abstract}
This paper investigates the existence of positive solutions to a two-point boundary value problem (BVP) for singular fractional differential equations in Banach space and presents a number of new results. First, by constructing a novel cone and using the fixed point index theory, a sufficient condition is established for the existence of at least two positive solutions to the approximate problem of the considered singular BVP. Second, using Ascoli-Arzela theorem, a sufficient condition is obtained for the existence of at least two positive solutions to the considered singular BVP from the convergent subsequence of the approximate problem. Finally, an illustrative example is given to support the obtained new results.
\end{abstract}

\section{Introduction}

Fractional differential equations have been widely investigated recently due to its wide applications [1-3] in biology, physics, medicine, control theory, and so forth. As a matter of fact, fractional derivatives provide a more excellent tool for the description of memory and hereditary properties of various materials and processes than integer derivatives. As an important issue for the theory of fractional differential equations, the existence of positive solutions to kinds of boundary value problems (BVPs) has attracted many scholars' attention, and lots of excellent results have been obtained [4-11] by means of fixed point theorems, upper and lower solutions technique, and so forth.

It is noted that as a special class of fractional differential equations, the singular fractional differential equations with kinds of boundary values have been studied in a series of recent works [7, 12, 13]. In [7], Jiang et al. studied a singular nonlinear semipositone fractional differential system with coupled boundary conditions and presented some sufficient conditions for the existence of a positive solution by using the fixed point theory in cone and constructing some available integral operators together with approximating technique. Zhang et al. [13] considered a class of two-point BVP for singular fractional differential equations with a negatively perturbed term and established some results on the multiplicity of positive solutions by using the approximating technique. In [12], Agarwal et al. investigated the existence of positive solutions for a two-point singular fractional boundary value problem and proposed some existence criteria by using sequential techniques. It should be pointed out that the nonlinearities of $[7,13]$ are singular at $t=0,1$, while the nonlinearity of [12] is singular at $x=0$. To our best knowledge, there are fewer results on two-point BVPs for singular fractional differential equations with the nonlinearity being singular at both $t=0,1$ and $x=0$. Motivated by this, we consider the following two-point BVP of singular fractional differential equations in Banach space:

$$
\begin{gathered}
D_{0^{+}}^{\beta} x(t)+f(t, x(t))=\theta, \quad t \in J, \\
x(0)=x(1)=\theta,
\end{gathered}
$$

where $1<\beta \leq 2$ is a real number, $J=[0,1], f: J \times \mathbf{E} \rightarrow$ $\mathbf{E}$ is continuous, $\theta$ denotes the null element in the Banach space $\mathbf{E}$ with the norm $\|\cdot\|, D_{0^{+}}^{\beta}$ is the standard RiemannLiouville fractional derivative, and $f(t, x)$ may be singular at $t=0,1$ and $x=\theta$. Firstly, we establish a sufficient condition for the existence of at least two positive solutions to the approximate problem of BVP (1) by constructing a novel cone and using the fixed point index theory. Secondly, using 
Ascoli-Arzela theorem, we obtained a sufficient condition for the existence of at least two positive solutions to BVP (1) from the convergent subsequence of the approximate problem. Finally, we give an illustrative example to support the obtained new results.

The main features of this paper are as follows. (i) A class of fractional-order two-point boundary value problems with the nonlinearity being singular at both $t=0,1$ and $x=\theta$ is firstly studied in this paper, which generalizes the existing singular fractional differential equations [7, 12, 13] and has wider applications. (ii) A sequential-based method is proposed for singular fractional differential equations with the nonlinearity being singular at both $t=0,1$ and $x=0$, which enriches the theory of fractional differential equations.

The rest of this paper is organized as follows. Section 2 contains the definition of Riemann-Liouville fractional derivative and some notation. The main results are presented in Section 3, which is followed by an illustrative example in Section 4.

\section{Preliminaries}

We first recall some well-known results about RiemannLiouville derivative. For details, please refer to $[14,15]$ and the references therein.

Definition 1. The Riemann-Liouville fractional integral of order $\beta>0$ of a function $y:(0, \infty) \rightarrow R$ is given by

$$
I_{0+}^{\beta} y(t)=\frac{1}{\Gamma(\beta)} \int_{0}^{t}(t-s)^{\beta-1} y(s) d s,
$$

provided the right side is pointwise defined on $(0, \infty)$.

Definition 2. The Riemann-Liouville fractional derivative of order $\beta>0$ of a continuous function $y:(0, \infty) \rightarrow R$ is given by

$$
D_{0+}^{\beta} y(t)=\frac{1}{\Gamma(n-\beta)}\left(\frac{d}{d t}\right)^{n} \int_{0}^{t} \frac{y(s)}{(t-s)^{\beta-n+1}} d s,
$$

where $n$ is the smallest integer greater than or equal to $\beta$, provided that the right side is pointwise defined on $(0, \infty)$.

One can easily obtain the following properties from the definition of Riemann-Liouville derivative.

Proposition 3 (see [15]). Let $\beta>0$; if one assumes that $u \in C(0,1) \cap L(0,1)$, then, the fractional differential equation $D_{0+}^{\beta} u(t)=0$ has $u(t)=C_{1} t^{\alpha-1}+C_{2} t^{\beta-2}+\cdots+C_{n} t^{\beta-n}$, $C_{i} \in R, i=1,2, \ldots, n$ as unique solutions, where $n$ is the smallest integer greater than or equal to $\beta$.

Proposition 4 (see [15]). Assume that $u \in C(0,1) \cap L(0,1)$ with a fractional derivative of order $\beta>0$ that belongs to $C(0,1) \cap L(0,1)$. Then,

$$
I_{0+}^{\beta} D_{0+}^{\beta} u(t)=u(t)+C_{1} t^{\beta-1}+C_{2} t^{\beta-2}+\cdots+C_{n} t^{\beta-n},
$$

for some $C_{i} \in R, i=1,2, \ldots, n$, where $n$ is the smallest integer greater than or equal to $\beta$.
The following lemmas will be used in the proof of the main results.

Lemma 5 (see [16]). If $S \subset C[J, \mathbf{E}]$ is bounded and equicontinuous, then

$$
\alpha_{c}(S)=\sup _{t \in J}(\alpha S(t)),
$$

where $\alpha(\cdot)$ and $\alpha_{c}(\cdot)$ denote the Kuratowski noncompactness measure of bounded sets in $\mathbf{E}$ and $C[J, \mathbf{E}]$, respectively, $S(t)=$ $\{x(t): x \in S\}(t \in J)$, and $C[J, \mathbf{E}]$ is the Banach space of all continuous functions $x: J \rightarrow \mathbf{E}$ with the norm $\|x\|_{c}=$ $\max _{t \in J}\|x(t)\|$.

Lemma 6 (see [16]). Let $P$ be a cone in $\mathbf{E}$, and let $P_{r}=\{x \in P$ : $\|x\|<r\}$. Let $F: P_{r} \rightarrow P$ be a strict set contraction. Assume that there exist $a u_{0} \in P$ and $u_{0} \neq \theta$ such that $x-F x \neq \lambda u_{0}$ for any $x \in \partial P_{r}$ and $\lambda \geq 0$. Then $i\left(F, P_{r}, P\right)=0$.

Lemma 7 (see [16]). Let $V=\left\{x_{n}\right\} \in L[J, \mathbf{E}]$, and there exists a $g \in L\left[J, R^{+}\right]$such that $\left\|x_{n}(t)\right\| \leq g(t)$, a.e. $t \in J$ for all $x_{n} \in V$; then $\alpha\left(\left\{\int_{a}^{t} x_{n}(s) d s: n \in N\right\}\right) \leq 2 \int_{a}^{t} \alpha(V(s)) d s, t \in J$.

Lemma 8 (Ascoli-Arzela theorem [16]). H $\subset \quad C[J, \mathbf{E}]$ is relative compact if and only if $H$ is equicontinuous, and for any $t \in J, H(t)$ is a relatively compact set in $\mathbf{E}$.

Lemma 9 (see [17]). Let $\mathbf{B} \subseteq \mathbf{E}$ be bounded open set. $A: P \cap$ $\overline{\mathbf{B}} \rightarrow \mathbf{B}$ is condensing. If there exists $1>\mu>0$, such that $A x=\mu x$, where $x \in P \bigcap \partial \mathbf{B}$, then $i(A, P \bigcap \mathbf{B}, P)=1$.

\section{Main Results}

Let $P=\{x \in \mathbf{E}: x(t) \geq \theta, \forall t \in J\}$. Then, one can see that $P$ is a normal solid cone of E. Define $P_{d}=\{x \in P:\|x\|<d\}$ and $\overline{P_{d}}=\{x \in P:\|x\| \leq d\}$. Let $P^{*}$ denote the dual cone of $P$. We consider BVP (1) in $C[J, \mathbf{E}] . x$ is called a solution to BVP (1), if $x$ satisfies (1). In addition, we call $x(t)$ a positive solution to $\operatorname{BVP}(1)$, if $x(t)>\theta, \forall t \in J$.

For convenience, let us list the following assumptions.

$$
\begin{aligned}
& \text { (H1) } f \in C[(0,1) \times P \backslash\{\theta\}, P] \text { and } \\
& \left\|f\left(t, t^{\beta-2} x\right)\right\| \leq k(t)\|q(x)\|, \quad t \in(0,1), x \in P \backslash\{\theta\},
\end{aligned}
$$

where $k:(0,1) \rightarrow(0,+\infty), \int_{0}^{1} s(1-s)^{\beta-1} k(s) d s<$ $+\infty$, and $q: P \backslash\{\theta\} \rightarrow P$.

(H2) $1 / \Gamma(\beta) \int_{0}^{1} s(1-s)^{\beta-1} k(s) q\left[(\beta-1) s(1-s)^{\beta-1} r_{1}, R_{1}\right] d s<$ $+\infty$ for all $R_{1}>r_{1}>0$, and there exists $R>0$ such that $1 / \Gamma(\beta) \int_{0}^{1} s(1-s)^{\beta-1} k(s) q\left[(\beta-1) s(1-s)^{\beta-1} R, R+\right.$ 1]ds $<R$, where

$$
q\left[r_{1}, R_{1}\right]:=\sup _{x \in \bar{P}_{R_{1}} \backslash P_{r_{1}}}\|q(x)\|<+\infty, \quad R_{1}>r_{1}>0 \text {. }
$$

(H3) $f\left(t, t^{\beta-2} x\right)$ is uniformly continuous with respect to $t$ on $[\gamma, 1-\gamma] \times \bar{P}_{R_{1}} \backslash P_{r_{1}}$, where $\gamma \in(0,1 / 2)$ and $R_{1}>$ $r_{1}>0$. 
(H4) There exists a constant $L \geq 0$, such that

$$
\begin{aligned}
& \alpha\left(f\left(t, t^{\beta-2} D\right)\right) \leq L \alpha(D), \\
& \forall t \in(0,1), \quad D \subseteq \bar{P}_{R_{1}} \backslash P_{r_{1}},
\end{aligned}
$$

where $L<\min \left\{\Gamma(\beta) \beta^{\beta} / 2(\beta-1)^{\beta-1}, \Gamma(\beta) \beta(\beta+1) / 2\right\}$, and $R_{1}>r_{1}>0$.

(H5) There exists $\varphi^{*} \in P^{*}$ with $\left\|\varphi^{*}\right\|=1$ and $\varphi \in L[0,1]$, such that

$$
\lim _{\|x\| \rightarrow 0} \inf _{x \in P} \varphi^{*}\left(f\left(t, t^{\beta-2} x\right)\right) \geq \varphi(t)
$$

uniformly in $t \in(0,1)$, where $0<\int_{0}^{1} s(1-$ $s)^{\beta-1} \varphi(s) d s<+\infty$.

(H6) There exist $\psi^{*} \in P^{*}$ with $\left\|\psi^{*}\right\|=1$ and $[a, b] \subseteq(0,1)$, such that

$$
\lim _{\|x\| \rightarrow+\infty} \inf _{x \in P} \frac{\psi^{*}\left(f\left(t, t^{\beta-2} x\right)\right)}{\|x\|}=+\infty,
$$

uniformly on $t \in[a, b]$.

According to [18], BVP (1) is equivalent to

$$
x(t)=\int_{0}^{1} G(t, s) f(s, x(s)) d s,
$$

where

$$
G(t, s)= \begin{cases}\frac{[t(1-s)]^{\beta-1}-(t-s)^{\beta-1}}{\Gamma(\beta)}, & 0 \leq s \leq t \leq 1, \\ \frac{[t(1-s)]^{\beta-1}}{\Gamma(\beta)}, & 0 \leq t \leq s \leq 1 .\end{cases}
$$

Consider the operator $A$ associated with the singular boundary value problem (1), which is defined by

$$
(A x)(t)=\int_{0}^{1} G(t, s) f(s, x(s)) d s .
$$

Set $G^{*}(t, s)=t^{2-\beta} G(t, s)$. As in [19], we have

$$
\begin{gathered}
G^{*}(t, s) \geq(\beta-1) t(1-t) G^{*}(\tau, s), \\
G^{*}(t, s) \leq \frac{1}{\Gamma(\beta)} s(1-s)^{\beta-1} .
\end{gathered}
$$

Define

$$
\left(A^{*} x\right)(t)=\int_{0}^{1} G^{*}(t, s) f\left(s, t^{\beta-2} x(s)\right) .
$$

Then, one can see that $x(t)$ is a fixed point of the operator $A^{*}$ if and only if $t^{\beta-2} x(t)$ is a solution of BVP (1).

Choose $e \in P^{o}$ with $\|e\|=1$. We consider the following approximate problem of (15):

$$
\left(A_{n}^{*} x\right)(t)=\int_{0}^{1} G^{*}(t, s) f\left(s, t^{\beta-2}\left(x(s)+\frac{e}{n}\right)\right) d s, \quad n \in N .
$$

\section{Denote}

$Q=\{x \in C[J, P]: x(t) \geq(\beta-1) t(1-t) x(s), \forall t, s \in J\}$

It is easy to check that $Q$ is a cone in $C[J, \mathbf{E}]$. Let $Q_{r}=\{x \in$ $\mathrm{Q}:\|x\|<r\}$. By (H1) and (H2), we can conclude that

$$
\begin{aligned}
\left\|A^{*} x\right\| \leq \int_{0}^{1} & \frac{s(1-s)^{\beta-1}}{\Gamma(\alpha)} k(s) \\
& \times q\left[(\beta-1) s(1-s)^{\beta-1} r, r\right] d s<+\infty, \quad \forall x \in Q_{r},
\end{aligned}
$$

which implies that the operator $A^{*}$ is well defined. In addition, from the definition of $Q$, we can prove that

$$
\|x(t)\| \geq(\beta-1) t(1-t)\|x\|_{c}, \quad \forall x \in Q, t \in J .
$$

By (16), we have $\left(A_{n}^{*} x\right)(t) \geq(\beta-1) t(1-t)\left(A_{n}^{*} x\right)(s), \forall t, s \in J$. Thus $A_{n}^{*} Q \subset Q$.

In the following, we first investigate the existence of two positive solutions to the approximate problem (16) and then establish the existence criterion for the existence of two positive solutions to BVP (1) by using the sequential-based technique and Ascoli-Arzela theorem.

Lemma 10. Let (H1) and (H2) be satisfied. Then for any $r>0$, the operator $A_{n}^{*}$ is a continuous bounded operator from $\bar{Q}_{r}$ into Q.

Proof. By (H1), we can easily see that $A_{n}^{*}$ is bounded from $Q_{r}$ to $Q$.

Next, we prove that $A_{n}^{*}$ is continuous.

Let $\left\|x_{m}-x\right\|_{c} \rightarrow 0$ as $m \rightarrow+\infty \quad\left(x_{m}, x \in \bar{Q}_{r}\right)$. From (H1), we have

$$
\begin{aligned}
\left\|f\left(s,\left(x_{m}(s)+\frac{e}{n}\right) s^{\beta-2}\right)\right\| \\
\quad \leq k(s) q\left(x_{m}(s)+\frac{e}{n}\right) \leq k(s) q\left[\frac{1}{n}, r+\frac{1}{n}\right] .
\end{aligned}
$$

Hence

$$
\begin{aligned}
\left\|\left(A_{n}^{*} x_{m}\right)(t)\right\| & =\left\|\int_{0}^{1} G^{*}(t, s) f\left(s,\left(x_{m}(s)+\frac{e}{n}\right) s^{\beta-2}\right) d s\right\| \\
& \leq \frac{1}{\Gamma(\beta)} \int_{0}^{1} s(1-s)^{\beta-1} k(s) q\left[\frac{1}{n}, r+\frac{1}{n}\right] d s \\
& <+\infty
\end{aligned}
$$

which together with the dominated convergence theorem imply that

$$
\lim _{m \rightarrow+\infty}\left(A_{n}^{*} x_{m}\right)(t)=\left(A_{n}^{*} x\right)(t) .
$$

We now show that

$$
\lim _{m \rightarrow+\infty}\left\|A_{n}^{*} x_{m}-A_{n}^{*} x\right\|_{c}=0 .
$$


In fact, if (23) is not true, then there exist a positive number $\varepsilon_{0}$ and a subsequence $\left\{x_{m_{i}}\right\} \subset\left\{x_{m}\right\}$ such that

$$
\left\|A_{n}^{*} x_{m_{i}}-A_{n}^{*} x\right\|_{c} \geq \varepsilon_{0}
$$

Since $\left\{A_{n}^{*} x_{m}\right\}$ is relatively compact, there is a subsequence of $\left\{A_{n}^{*} x_{m_{i}}\right\}$ which converges to some $y \in Q$. Without loss of generality, we assume that $\left\{A_{n}^{*} x_{m_{i}}\right\}$ itself converges to $y$, that is,

$$
\lim _{i \rightarrow \infty}\left\|A_{n}^{*} x_{m_{i}}-y\right\|_{c}=0
$$

By virtue of (22) and (25), we have $y=A_{n}^{*} x$, which contradicts with (24). Hence (23) holds, and the continuity of $A_{n}^{*}$ is proved.

Lemma 11. Let (H1)-(H4) be satisfied. Then for any $r>0$, the operator $A_{n}^{*}$ is a strict set contraction from $Q_{r}$ to $Q$.

Proof. By virtue of Lemma 10, we know that $A_{n}^{*} S$ is bounded and equicontinuous on $J$. Thus, from Lemma 5 , one can see that

$$
\alpha_{c}\left(A_{n}^{*} S\right)=\sup _{t \in J} \alpha\left(\left(A_{n}^{*} S\right)(t)\right), \quad r>0, S \subseteq Q_{r}
$$

where $A_{n}^{*} S(t)=\left\{\left(A_{n}^{*} x\right)(t): x \in S\right\}, t \in J$.

Set

$$
\begin{array}{r}
D_{\delta}=\left\{\int_{\delta}^{1-\delta} G^{*}(t, s) f\left(s,\left(x(s)+\frac{e}{n}\right) s^{\beta-2}\right) d s: x \in S\right\}, \\
\delta \in\left(0, \frac{1}{2}\right) .
\end{array}
$$

Based on (H1) and (H2), we have

$$
\begin{aligned}
& \| \int_{\delta}^{1-\delta} G^{*}(t, s) f\left(s,\left(x(s)+\frac{e}{n}\right) s^{\beta-2}\right) d s \\
& \quad-\int_{0}^{1} G^{*}(t, s) f\left(s,\left(x(s)+\frac{e}{n}\right) s^{\beta-2}\right) d s \| \\
& \leq c\left(\int_{0}^{\delta} s(1-s)^{\beta-1} k(s) d s\right. \\
& \left.\quad+\int_{1}^{1-\delta} s(1-s)^{\beta-1} k(s) d s\right), \quad x \in S, \delta \in\left(0, \frac{1}{2}\right),
\end{aligned}
$$

where $c=q[1 / n, r+(1 / n)]$. Hence

$$
d_{H}\left(D_{\delta}, A_{n}^{*} S\right) \longrightarrow 0, \quad \delta \longrightarrow 0^{+},
$$

where $d_{H}(\cdot, \cdot)$ denotes the Hausdorff metrics, which implies that

$$
\alpha\left(A_{n}^{*} S\right)=\lim _{\delta \rightarrow 0^{+}} \alpha\left(D_{\delta}\right) .
$$

Since

$$
\begin{aligned}
& \int_{\delta}^{1-\delta} G^{*}(t, s) f\left(s, s^{\beta-2}\left(x(s)+\frac{e}{n}\right)\right) d s \\
& \in(1-2 \delta) \\
& \quad \times \overline{c o}\left\{G^{*}(t, s) f\left(s, s^{\beta-2}\left(x(s)+\frac{e}{n}\right)\right): s \in[\delta, 1-\delta]\right\},
\end{aligned}
$$

by Lemmas 5 and 7, we have

$$
\begin{aligned}
& \alpha\left(D_{\delta}\right) \\
& =\alpha\left(\left\{\int_{\delta}^{1-\delta} G^{*}(t, s) f\left(s, s^{\beta-2}\left(x(s)+\frac{e}{n}\right)\right) d s, x \in S\right\}\right) \\
& \leq(1-2 \delta) \\
& \times \alpha\left(\overline { \mathrm { CO } } \left\{G^{*}(t, s)\right.\right. \\
& \left.\left.\times f\left(s,\left(x(s)+\frac{e}{n}\right) s^{\beta-2}\right): s \in[\delta, 1-\delta], x \in S\right\}\right) \\
& \leq \alpha\left(\left\{G^{*}(t, s)\right.\right. \\
& \left.\left.\times f\left(s,\left(x(s)+\frac{e}{n}\right) s^{\beta-2}\right): s \in[\delta, 1-\delta], x \in S\right\}\right) \\
& \leq \frac{1}{\Gamma(\beta)} \frac{(\beta-1)^{\beta-1}}{\beta^{\beta}} \\
& \times \alpha\left(\left\{f\left(s,\left(x(s)+\frac{e}{n}\right) s^{\beta-2}\right): s \in[\delta, 1-\delta], x \in S\right\}\right) \\
& \leq \frac{1}{\Gamma(\beta)} L \frac{(\beta-1)^{\beta-1}}{\beta^{\beta}} \alpha\left(S\left(I_{\delta}\right)\right) \\
& \leq \frac{1}{\Gamma(\beta)} 2 L \frac{(\beta-1)^{\beta-1}}{\beta^{\beta}} \alpha_{c}(S),
\end{aligned}
$$

where $I_{\delta}=[\delta, 1-\delta]$. By $(\mathrm{H} 4)$, we can obtain

$$
\begin{aligned}
\alpha\left(A_{n} S\right) & =\lim _{\delta \rightarrow 0^{+}} \alpha\left(D_{\delta}\right) \\
& \leq \frac{1}{\Gamma(\beta)} \frac{(\beta-1)^{\beta-1}}{\beta^{\beta}} 2 L \alpha_{c}(S)<\alpha_{c}(S) .
\end{aligned}
$$

Consequently, the operator $A_{n}$ is a strict set contraction from $Q_{r}$ into $Q$.

Theorem 12. Let (H1)-(H6) hold. Then, there exists $r \in(0, R)$ such that the operator $A_{n}^{*}$ has two fixed points in $Q_{R} \backslash \bar{Q}_{r}$ and $Q \backslash$ $\bar{Q}_{R}$, respectively, for arbitrary sufficiently large positive integer $n$, where $R$ is given in (H2). 
Proof. For any given $t \in(0,1)$, we have $\int_{0}^{1} G^{*}(t, s) \varphi(s) d s \leq$ $\int_{0}^{1} s(1-s)^{\beta-1} \varphi(s) d s$. Since $\int_{0}^{1} G^{*}(t, s) \varphi(s) d s>0$, there exists a $\varepsilon^{\prime}>0$ such that

$$
r^{\prime}=\int_{0}^{1} \int_{0}^{1} G^{*}(t, s)\left(\varphi(s)-\varepsilon^{\prime}\right) d s d t>0 .
$$

Then by (H5), there exists $r^{\prime \prime} \in(0, R)$ such that

$$
\varphi^{*}\left(f\left(t, t^{\beta-2} x(t)\right)\right) \geq \varphi(t)-\varepsilon^{\prime}, \quad \forall t \in(0,1)
$$

holds for $x \in Q$ and $\|x\|_{c} \leq r^{\prime \prime}$. Letting $0<r<l:=\min \left\{r^{\prime}\right.$, $\left.r^{\prime \prime}\right\}$, we prove that $x-A_{n}^{*} x \neq \lambda$ e for any $x \in \partial Q_{r}, \lambda \geq 0$ as $n>1 /(l-r)$. In fact, suppose that this is false. Then there exist $\lambda>0, x \in \partial Q_{r}$ such that $x-A_{n}^{*} x=\lambda e$. It is obvious that

$$
\begin{aligned}
x(t) & =\left(A_{n}^{*} x\right)(t)+\lambda e \geq\left(A_{n}^{*} x\right)(t) \\
& =\int_{0}^{1} G^{*}(t, s) f\left(s,\left(x(s)+\frac{e}{n}\right) s^{\beta-2}\right) d s .
\end{aligned}
$$

Thus

$$
\begin{aligned}
& \varphi^{*}(x(t)) \\
& \quad \geq \int_{0}^{1} G^{*}(t, s) \varphi^{*}\left(f\left(s,\left(x(s)+\frac{e}{n}\right) s^{\beta-2}\right)\right) d s \\
& \quad \geq \int_{0}^{1} G^{*}(t, s)\left(\varphi(s)-\varepsilon^{\prime}\right) d s .
\end{aligned}
$$

This implies that

$$
\begin{aligned}
& \int_{0}^{1} \varphi^{*}(x(t)) d t \\
& \quad \geq \int_{0}^{1} \int_{0}^{1} G^{*}(t, s)\left(\varphi(s)-\varepsilon^{\prime}\right) d s d t=r^{\prime}>r,
\end{aligned}
$$

which is in contradiction with

$$
\left|\varphi^{*}(x(t))\right| \leq\|x(t)\| \leq\|x\|_{\mathcal{c}}=r, \quad t \in J .
$$

Combining with Lemma 6, we obtain that

$$
i\left(A_{n}^{*}, Q_{r}, Q\right)=0 .
$$

Now, we show that $i\left(A_{n}^{*}, Q_{R}, Q\right)=1$. By Lemma 9, we need only to prove that $A_{n}^{*} x \neq \lambda x$ for $x \in \partial Q_{R}$ and $\lambda \geq 1$. In fact, if there exist $x \in \partial Q_{R}$ and some $\lambda \geq 1$ such that $A_{n}^{*} x=$ $\lambda x$, then

$$
\begin{aligned}
x(t) & =\frac{1}{\lambda} \int_{0}^{1} G^{*}(t, s) f\left(s,\left(x(s)+\frac{e}{n}\right) s^{\beta-2}\right) d s \\
& \leq \int_{0}^{1} G^{*}(t, s) f\left(s,\left(x(s)+\frac{e}{n}\right) s^{\beta-2}\right) d s \\
& \leq \frac{1}{\Gamma(\beta)} \int_{0}^{1} s(1-s)^{\beta-1} f\left(s,\left(x(s)+\frac{e}{n}\right) s^{\beta-2}\right) d s .
\end{aligned}
$$

Therefore, by (H1) and (H2), we have

$$
\begin{aligned}
& R=\|x\|_{c} \\
& <\frac{1}{\Gamma(\beta)} \int_{0}^{1} s(1-s)^{\beta-1} k(s)\left\|q\left(x(s)+\frac{e}{n}\right)\right\| d s \\
& <\frac{1}{\Gamma(\beta)} \int_{0}^{1} s(1-s)^{\beta-1} k(s) \\
& \quad \times q\left[(\beta-1) s(1-s)^{\beta-1} R, R+1\right] d s
\end{aligned}
$$

$<R$,

which is a contradiction. Consequently,

$$
i\left(A_{n}^{*}, Q_{R}, Q\right)=1 .
$$

In the following, we choose

$$
R^{\prime}>\left((\beta-1) a(1-b) \int_{a}^{b} \max _{t \in J} G^{*}(t, s) d s\right)^{-1} .
$$

By (H6), there exists $M>R$ such that $\varphi^{*}\left(f\left(t, t^{\beta-2} x\right)\right) \geq R^{\prime}\|x\|$ holds for $x>M$.

Let

$$
\begin{aligned}
\bar{R}=\max & \left\{\frac{M+1}{(\beta-1) a(1-b)},\right. \\
& \left.1+\frac{1}{(\beta-1) R^{\prime} a(1-b) \max _{t \in J} \int_{a}^{b} G^{*}(t, s) d s-1}\right\} .
\end{aligned}
$$

We now claim that $x-A_{n}^{*} x \neq \lambda$ e for $x \in \partial Q_{\bar{R}}$ and $\lambda \geq 0$. As a matter of fact, if this is not true, then there exists $\lambda>0$ such that $x-A_{n}^{*} x=\lambda e$, that is, $x(t)>A_{n}^{*} x(t)$, which implies that

$$
\begin{aligned}
\bar{R} & \geq \varphi^{*}(x(t)) \\
& \geq \varphi^{*}\left(\left(A_{n}^{*} x\right)(t)\right) \\
& \geq \int_{0}^{1} G^{*}(t, s) \varphi^{*}\left(f\left(s,\left(x(s)+\frac{e}{n}\right) s^{\beta-2}\right)\right) d s \\
& \geq \int_{0}^{1} G^{*}(t, s) R^{\prime}((\beta-1) a(1-b)(\|x\|-1)) d s \\
& \geq(\beta-1) a(1-b) R^{\prime}(\bar{R}-1) \int_{a}^{b} G^{*}(t, s) d s \\
& >\bar{R},
\end{aligned}
$$

which is a contradiction. Therefore, by Lemma 6, we can obtain that

$$
i\left(A_{n}^{*}, Q_{\bar{R}}, Q\right)=0 .
$$


This together with (40), (43), and (47) implies that

$$
\begin{gathered}
i\left(A_{n}^{*}, Q_{R} \backslash \bar{Q}_{r}, Q\right)=i\left(A_{n}^{*}, Q_{R}, Q\right)-i\left(A_{n}^{*}, Q_{r}, Q\right)=1, \\
i\left(A_{n}^{*}, Q_{\bar{R}} \backslash \bar{Q}_{R}, Q\right)=i\left(A_{n}^{*}, Q_{\bar{R}}, Q\right)-i\left(A_{n}^{*}, Q_{R}, Q\right)=-1 .
\end{gathered}
$$

Thus, there exist $x \in Q_{R} \backslash \bar{Q}_{r}$ and $y \in Q_{\bar{R}} \backslash \bar{Q}_{R}$ such that $A_{n}^{*} x=x$ and $A_{n}^{*} y=y$. This completes the proof.

Theorem 13. Let (H1)-(H6) be satisfied. Then BVP (1) has at least two positive solutions in $C[J, \mathbf{E}]$.

Proof. From Theorem 12, there exists an integer $n_{0}>0$, such that the operator $A_{n}^{*}, n \geq n_{0}$ has two fixed points in $x_{n} \in Q_{R} \backslash$ $\bar{Q}_{r}$ and $y_{n} \in Q \backslash \bar{Q}_{R}$. Denote $D:=\left\{x_{n}: n \geq n_{0}\right\}$. Obviously $D$ is uniformly bounded. Next, we show that $D$ is equicontinuous.

Firstly, we prove that

$$
\begin{aligned}
& \lim _{t \rightarrow 0^{+}} \int_{0}^{1} G^{*}(t, s) f\left(s,\left(x(s)+\frac{e}{n}\right) s^{\beta-2}\right) d s=0, \\
& \lim _{t \rightarrow 1^{-}} \int_{0}^{1} G^{*}(t, s) f\left(s,\left(x(s)+\frac{e}{n}\right) s^{\beta-2}\right) d s=0 .
\end{aligned}
$$

On one hand, by the absolute continuity of integration [19], for any $\varepsilon>0$, there exists $\delta_{1}>0$ such that $\int_{0}^{t}(1 / \Gamma(\beta)) s(1-s)^{\beta-1} f\left(s,(x(s)+e / n) s^{\beta-2}\right) d s<\varepsilon / 2, \forall 0<t \leq$ $\delta_{1}$.

On the other hand, from $(\mathrm{H} 2)$, we set

$$
\bar{M}:=\int_{\delta_{1}}^{1} \frac{s(1-s)^{\beta-1}}{\Gamma(\beta)} k(s) q\left[(\beta-1) s(1-s)^{\beta-1} r, R\right] d s,
$$

and $\delta=\min \left\{\delta_{1}, \delta_{1} \varepsilon / \bar{M}\right\}$. For $0<t<\delta$, we have

$$
\begin{aligned}
\int_{0}^{1} & G^{*}(t, s) f\left(s,\left(x(s)+\frac{e}{n}\right) s^{\beta-2}\right) d s \\
= & \int_{0}^{\delta_{1}} G^{*}(t, s) f\left(s,\left(x(s)+\frac{e}{n}\right) s^{\beta-2}\right) d s \\
& +\int_{\delta_{1}}^{1} G^{*}(t, s) f\left(s,\left(x(s)+\frac{e}{n}\right) s^{\beta-2}\right) d s \\
< & \frac{\varepsilon}{2}+\int_{\delta_{1}}^{1} \frac{t(1-s)^{\beta-1}}{\Gamma(\beta)} f\left(s,\left(x(s)+\frac{e}{n}\right) s^{\beta-2}\right) d s \\
\leq & \frac{\varepsilon}{2}+\frac{t}{\delta_{1}} \int_{\delta_{1}}^{1} \frac{s(1-s)^{\beta-1}}{\Gamma(\beta)} k(s) q\left(x(s)+\frac{e}{n}\right) d s \\
\leq & \frac{\varepsilon}{2}+\frac{t}{\delta_{1}} \int_{\delta_{1}}^{1} \frac{s(1-s)^{\beta-1}}{\Gamma(\beta)} k(s) q\left[(\beta-1) s(1-s)^{\beta-1} r, R\right] d s \\
\leq & \varepsilon,
\end{aligned}
$$

which implies that (49) holds. Similarly, one can prove that (50) holds.
Next, we show that $D$ is equicontinuous for $t \in[\gamma, 1-\gamma]$, $0<\gamma<1 / 2$.

From (H3), we define

$$
\widehat{M}=\max _{t \in[\gamma, 1-\gamma]} f\left(t,\left(x(t)+\frac{e}{n}\right) t^{\beta-2}\right) .
$$

By the absolute continuity of integration, for $\gamma \in(0,1 / 2)$ and the previous $\varepsilon>0$, there exists $0<\delta_{2}<\gamma$ such that

$$
\left|\int_{0}^{\delta_{2}} \frac{s(1-s)^{\beta-1}}{\Gamma(\beta)} f\left(s,\left(x(s)+\frac{e}{n}\right) s^{\beta-2}\right) d s\right|<\frac{\varepsilon}{6} .
$$

Since $\left(\left(t_{2}(1-s)^{\beta-1}-\left(t_{2}-s\right)^{\beta-1} t_{2}^{2-\beta}\right) / \Gamma(\beta)\right) f(s,(x(s)+$ $\left.e / n) s^{\beta-2}\right)$ is bounded for $s \in\left[t_{1}, t_{2}\right]$, there exists $\delta_{3}>0$, such that

$$
\begin{aligned}
& \left|\int_{t_{1}}^{t_{2}} \frac{t_{2}(1-s)^{\beta-1}-\left(t_{2}-s\right)^{\beta-1} t_{2}^{2-\beta}}{\Gamma(\beta)} f\left(s,\left(x(s)+\frac{e}{n}\right) s^{\beta-2}\right) d s\right| \\
& \quad<\frac{\varepsilon}{6}, \quad\left|t_{2}-t_{1}\right|<\delta_{3} .
\end{aligned}
$$

For $\left|t_{2}-t_{1}\right|<\delta_{4}:=\gamma \varepsilon /\left(6 \int_{0}^{1}\left(s(1-s)^{\beta-1} / \Gamma(\beta)\right) k(s) q[(\beta-\right.$ 1) $\left.\left.s(1-s)^{\beta-1} r, R\right] d s\right)$, one can see that

$$
\left|\int_{t_{2}}^{1} \frac{\left(t_{2}-t_{1}\right)(1-s)^{\beta-1}}{\Gamma(\beta)} f\left(s,\left(x(s)+\frac{e}{n}\right) s^{\beta-2}\right) d s\right|<\frac{\varepsilon}{6} .
$$

Similarly, for $\left|t_{2}-t_{1}\right|<\delta_{5}:=\delta_{2} \varepsilon /\left(6 \int_{0}^{1}(s(1-\right.$ $\left.\left.s)^{\beta-1} / \Gamma(\beta)\right) k(s) q\left[(\beta-1) s(1-s)^{\beta-1} r, R\right] d s\right)$, we have

$$
\left|\int_{\delta_{2}}^{t_{1}} \frac{\left(t_{2}-t_{1}\right)(1-s)^{\beta-1}}{\Gamma(\beta)} f\left(s,\left(x(s)+\frac{e}{n}\right) s^{\beta-2}\right) d s\right|<\frac{\varepsilon}{6} .
$$

Since

$$
\begin{aligned}
& \mid \int_{\delta_{2}}^{t_{1}} \frac{t_{2}^{2-\beta}\left(t_{2}-s\right)^{\beta-1}-t_{1}^{2-\beta}\left(t_{1}-s\right)^{\beta-1}}{\Gamma(\beta)} \\
& \quad \times f\left(s,\left(x(s)+\frac{e}{n}\right) s^{\beta-2}\right) d s \mid \\
& \leq \mid \frac{1}{\delta_{2}\left(1-t_{1}\right)^{\beta-1}} \int_{\delta_{2}}^{t_{1}} \frac{t_{2}^{2-\beta}\left(t_{2}-s\right)^{\beta-1}-t_{1}^{2-\beta}\left(t_{1}-s\right)^{\beta-1}}{\Gamma(\beta)}
\end{aligned}
$$

$$
\times s(1-s)^{\beta-1} f\left(s,\left(x(s)+\frac{e}{n}\right) s^{\beta-2}\right) d s \mid,
$$


$f\left(t,(x(t)+e / n) t^{\beta-2}\right)$ is bounded on $t \in\left[\delta_{2}, t_{1}\right]$, and $\left(t_{2}^{2-\beta}\left(t_{2}-\right.\right.$ $\left.s)^{\beta-1}-t_{1}^{2-\beta}\left(t_{1}-s\right)^{\beta-1}\right) / \Gamma(\beta) \rightarrow 0$ as $t_{1} \rightarrow t_{2}$, there exists $\delta_{6}>0$ such that

$$
\begin{aligned}
& \mid \int_{\delta_{2}}^{t_{1}} \frac{t_{2}^{2-\beta}\left(t_{2}-s\right)^{\beta-1}-t_{1}^{2-\beta}\left(t_{1}-s\right)^{\beta-1}}{\Gamma(\beta)} \\
& \quad \times f\left(s,\left(x(s)+\frac{e}{n}\right) s^{\beta-2}\right) d s \mid \\
& \quad<\frac{\varepsilon}{6}, \quad\left|t_{2}-t_{1}\right|<\delta_{6} .
\end{aligned}
$$
we have

We choose $\delta^{\prime}=\min \left\{\delta_{i}: i=2,3,4,5,6\right\}$. For $\left|t_{2}-t_{1}\right|<\delta^{\prime}$,

$$
\begin{aligned}
& \left|x\left(t_{2}\right)-x\left(t_{1}\right)\right| \\
& =\mid \int_{0}^{1} G^{*}\left(t_{2}, s\right) f\left(s,\left(x(s)+\frac{e}{n}\right) s^{\beta-2}\right) d s \\
& -\int_{0}^{1} G^{*}\left(t_{1}, s\right) f\left(s,\left(x(s)+\frac{e}{n}\right) s^{\beta-2}\right) d s \mid \\
& \leq\left|\int_{0}^{\delta_{2}} G^{*}\left(t_{2}, s\right) f\left(s,\left(x(s)+\frac{e}{n}\right) s^{\beta-2}\right) d s\right| \\
& +\left|\int_{0}^{\delta_{2}} G^{*}\left(t_{1}, s\right) f\left(s,\left(x(s)+\frac{e}{n}\right) s^{\beta-2}\right) d s\right| \\
& +\mid \int_{\delta_{2}}^{t_{2}} G^{*}\left(t_{1}, s\right) f\left(s,\left(x(s)+\frac{e}{n}\right) s^{\beta-2}\right) d s \\
& +\int_{t_{2}}^{1} G^{*}\left(t_{2}, s\right) f\left(s,\left(x(s)+\frac{e}{n}\right) s^{\beta-2}\right) d s \\
& -\int_{\delta_{2}}^{t_{1}} G^{*}\left(t_{1}, s\right) f\left(s,\left(x(s)+\frac{e}{n}\right) s^{\beta-2}\right) d s \\
& -\int_{t_{1}}^{1} G^{*}\left(t_{1}, s\right) f\left(s,\left(x(s)+\frac{e}{n}\right) s^{\beta-2}\right) d s \mid \\
& \leq 2\left|\int_{0}^{\delta_{2}} \frac{s(1-s)^{\beta-1}}{\Gamma(\beta)} f\left(s,\left(x(s)+\frac{e}{n}\right) s^{\beta-2}\right) d s\right| \\
& +\mid \int_{\delta_{2}}^{t_{2}} \frac{t_{2}(1-s)^{\beta-1}-\left(t_{2}-s\right)^{\beta-1} t_{2}^{2-\beta}}{\Gamma(\beta)} \\
& \times f\left(s,\left(x(s)+\frac{e}{n}\right) s^{\beta-2}\right) d s \\
& +\left|\int_{t_{2}}^{t_{1}} \frac{t_{1}(1-s)^{\beta-1}}{\Gamma(\beta)} f\left(s,\left(x(s)+\frac{e}{n}\right) s^{\beta-2}\right) d s\right| \\
& +\left|\int_{t_{2}}^{t_{1}} \frac{\left(t_{2}-t_{1}\right)(1-s)^{\beta-1}}{\Gamma(\beta)} f\left(s,\left(x(s)+\frac{e}{n}\right) s^{\beta-2}\right) d s\right| \\
& +\left|\int_{\delta_{2}}^{t_{1}} \frac{\left(t_{2}-t_{1}\right)(1-s)^{\beta-1}}{\Gamma(\beta)} f\left(s,\left(x(s)+\frac{e}{n}\right) s^{\beta-2}\right) d s\right|
\end{aligned}
$$

$$
\begin{gathered}
+\mid \int_{\delta_{2}}^{t_{1}} \frac{\left(t_{2}^{2-\beta}\left(t_{2}-s\right)^{\beta-1}-t_{1}^{2-\beta}\left(t_{1}-s\right)^{\beta-1}\right)}{\Gamma(\beta)} \\
\quad \times f\left(s,\left(x(s)+\frac{e}{n}\right) s^{\beta-2}\right) d s \mid<\varepsilon .
\end{gathered}
$$

This together with (49) and (50) implies that $D$ is equicontinuous for $t \in[0,1]$.

Now, we show that $D(t)$ is relatively compact. By Lemma 7, we have

$\alpha(D(t))$

$$
\begin{aligned}
& \leq \alpha\left(\left\{\int_{0}^{1} G^{*}(t, s) f\left(s,\left(x_{n}(s)+\frac{e}{n}\right) s^{\beta-2} d s\right): n \geq n_{0}\right\}\right) \\
& \leq 2 \int_{0}^{1} G^{*}(t, s) \\
& \quad \times \alpha\left(\left\{f\left(s,\left(x_{n}(s)+\frac{e}{n}\right) s^{\beta-2}\right): n \geq n_{0}\right\}\right) d s \\
& \leq 2 L \int_{0}^{1} G^{*}(t, s) \alpha(D(s)) d s
\end{aligned}
$$

which together with Lemma 5 and (H4) implies that

$$
\alpha_{c}(D) \leq 2 L \frac{1}{\Gamma(\beta)}\left(\int_{0}^{1} s(1-s)^{\beta-1} d s\right) \alpha_{c}(D)
$$

Thus, $\alpha_{c}(D)=0$. It follows from Lemma 8 that there is a convergent subsequence of $\left\{x_{n}\right\}$. Without loss of generality, we assume that $\left\{x_{n}\right\}$ itself converges to some $x \in Q$. Then, the dominated convergence theorem and (16) imply that

$$
x(t)=\int_{0}^{1} G^{*}(t, s) f\left(s, s^{\beta-2} x(s)\right) d s .
$$

Thus, the singular BVP (1) has a positive solution $t^{\beta-2} x(t)$. Similarly, $\left\{y_{n}\right\}$ has a convergent subsequence, which converges to $y(t)$. Then, $t^{\beta-2} y(t)$ is also a positive solution to BVP (1).

Finally, we show $x \neq y$. We only need to prove that the operator $A^{*}$ has no fixed point in $\partial Q_{R}$.

In fact, if it is not true, then we assume that $z$ is a fixed point of the operator $A^{*}$ in $\partial Q_{R}$. Then

$$
z(t)=\int_{0}^{1} G^{*}(t, s) f\left(s, s^{\beta-2} z(s)\right) d s,
$$

with $(\beta-1) t(1-t)^{\beta-1} R \leq\|z(t)\| \leq R+1$. 
By $(\mathrm{H} 1)$ and $(\mathrm{H} 2)$, one can see that

$$
\begin{aligned}
R & =\max _{t \in J}\|z(t)\| \\
& \leq \int_{0}^{1} s(1-s)^{\beta-1} k(s) q\left[(\beta-1) s(1-s)^{\beta-1} R, R\right] d s \\
& \leq \int_{0}^{1} s(1-s)^{\beta-1} k(s) q\left[(\beta-1) s(1-s)^{\beta-1} R, R+1\right] d s \\
& <R,
\end{aligned}
$$

which is a contradiction. The proof of this theorem is completed.

Remark 14. It is noted that the singularity of $f(t, x)$ at $t=0,1$ is overcome by (H2), while the singularity at $x=\theta$ is handled by solving the approximate problem and using the sequentialbased technique.

\section{Example}

Consider the following BVP:

$$
\begin{aligned}
- & D_{0^{+}}^{3 / 2} x_{n}(t) \\
= & \frac{1}{2 \pi \sqrt{t(1-t)}} \\
& \times\left(\frac{1}{\left|\sum_{i=1}^{m} x_{i}(t)\right| t^{1 / 2}}+\sqrt{t} x_{n+1}(t)+t x_{n+2}^{2}(t)\right), \\
& \quad t \in(0,1), \\
& x_{n}(0)=x_{n}(1)=0, \quad n=1,2, \ldots, m,
\end{aligned}
$$

where $x_{m+n}=x_{n}$. Then, $\operatorname{BVP}(66)$ has at least two positive solutions.

Proof. We consider the problem (66) in $m$-dimensional Euclidean space $R^{m}=\left\{x=\left(x_{1}, x_{2}, \ldots, x_{m}\right): x_{n} \in R, n=\right.$ $1,2, \ldots, m\}$ with the norm $\|x\|=\sum_{n=1}^{m}\left|x_{n}\right|$. Then, BVP (66) has the form of (1) with

$$
\begin{gathered}
x(t)=\left(x_{1}(t), x_{2}(t), \ldots x_{m}(t)\right) \\
f_{n}(t, x)=\frac{1}{2 \pi \sqrt{t(1-t)}}\left(\frac{1}{\left|\sum_{i=1}^{m} x_{i}\right| t^{1 / 2}}+\sqrt{t} x_{n+1}+t x_{n+2}^{2}\right) .
\end{gathered}
$$

It is easy to see that $f(t, x)$ is singular at $t=0,1$ and $x=\theta$. Set $k(t)=1 /(2 \pi \sqrt{t(1-t)})$ and $q(x)=\left(1 /\left|\sum_{i=1}^{m} x_{i}\right|\right)+x_{n+1}+$ $x_{n+2}^{2}$. One can easily see that (H1) holds.

We choose $R=1$ and $\varphi^{*}=\phi^{*}=(1,1, \ldots, 1)$. Considering that

$$
\int_{0}^{1} \sqrt{s(1-s)} d s=\frac{\pi}{8}
$$

we can easily check that (H2)-(H6) hold. From Theorem 13, BVP (66) has at least two positive solutions $x=\left(x_{1}, x_{2}, \ldots x_{m}\right)$ and $\left(y_{1}, y_{2}, \ldots y_{m}\right)$ which satisfy $0<\|x\|<1<\|y\|<+\infty$.

\section{Acknowledgments}

The authors wish to thank referees and Dr. Haitao Li for their valuable suggestions. The project was supported by the National Natural Science Foundation of China (11171192), Graduate Educational Innovation Foundation of Shandong Province (SDYY1005), and the Promotive Research Fund for Excellent Young and Middle-Aged Scientists of Shandong Province (BS2010SF025).

\section{References}

[1] A. A. Kilbas and J. J. Trujillo, "Differential equations of fractional order: methods, results and problems. I," Applicable Analysis, vol. 78, no. 1-2, pp. 153-192, 2001.

[2] A. A. Kilbas and J. J. Trujillo, "Differential equations of fractional order: methods, results and problems. II," Applicable Analysis, vol. 81, no. 2, pp. 435-493, 2002.

[3] I. Podlubny, Fractional Differential Equations, Mathematics in Science and Engineering, Academic Press, New York, NY, USA, 1999.

[4] R. P. Agarwal and D. O’Regan, "Singular boundary value problems," Nonlinear Analysis. Theory, Methods \& Applications A, vol. 27, pp. 645-656, 1996.

[5] R. P. Agarwal and D. O’Regan, "Nonlinear superlinear singular and nonsingular second order boundary value problems," Journal of Differential Equations, vol. 143, no. 1, pp. 60-95, 1998.

[6] H. H. Alsulami, S. K. Ntouyas, and B. Ahmad, "Existence results for a Riemann-Liouvilletype fractional multivalued problem with integral boundary conditions," Journal of Function Spaces and Applications, vol. 2013, Article ID 676045, 7 pages, 2013.

[7] J. Jiang, L. Liu, and Y. Wu, "Positive solutions to singular fractional differential system with coupled boundary conditions," Communications in Nonlinear Science and Numerical Simulation, vol. 18, no. 11, pp. 3061-3074, 2013.

[8] H. Li, X. Kong, and C. Yu, "Existence of three non-negative solutions for a three-point boundary-value problem of nonlinear fractional differential equations," Electronic Journal of Differential Equations, vol. 88, pp. 12-1, 2012.

[9] A. Shi and S. Zhang, "Upper and lower solutions method and a fractional differential equation boundary value problem," Electronic Journal of Qualitative Theory of Differential Equations, vol. 30, pp. 1-13, 2009.

[10] Z. L. Wei, "Positive solutions of singular boundary value problems for negative exponent Emden-Fowler equations," Acta Mathematica Sinica. Chinese Series, vol. 41, no. 3, pp. 655-662, 1998 (Chinese).

[11] S. Zhang, "Positive solutions for boundary-value problems of nonlinear fractional differential equations," Electronic Journal of Differential Equations, vol. 36, pp. 1-12, 2006.

[12] R. P. Agarwal, D. O’Regan, and S. Staněk, "Positive solutions for Dirichlet problems of singular nonlinear fractional differential equations," Journal of Mathematical Analysis and Applications, vol. 371, no. 1, pp. 57-68, 2010. 
[13] X. Zhang, L. Liu, and Y. Wu, "Multiple positive solutions of a singular fractional differential equation with negatively perturbed term," Mathematical and Computer Modelling, vol. 55, no. 3-4, pp. 1263-1274, 2012.

[14] K. B. Oldham and J. Spanier, The Fractional Calculus, Academic Press, New York, NY, USA, 1974.

[15] S. G. Samko, A. A. Kilbas, and O. I. Marichev, Fractional Integrals and Derivatives (Theory and Applications), Gordon and Breach, Yverdon, Switzerland, 1993.

[16] D. Guo, V. Lakshmikantham, and X. Liu, Nonlinear Integral Equations in Abstract Spaces, vol. 373, Kluwer Academic Publishers, Dordrecht, The Netherlands, 1996.

[17] D. Guo, Nonlinera Function Analysis, Shandong Science and Technology Publishing House, Jinan, China, 1985.

[18] Z. Bai and H. Lü, "Positive solutions for boundary value problem of nonlinear fractional differential equation," Journal of Mathematical Analysis and Applications, vol. 311, no. 2, pp. 495-505, 2005.

[19] D. Jiang and C. Yuan, "The positive properties of the Green function for Dirichlet-type boundary value problems of nonlinear fractional differential equations and its application," Nonlinear Analysis. Theory, Methods \& Applications A, vol. 72, no. 2, pp. 710-719, 2010. 


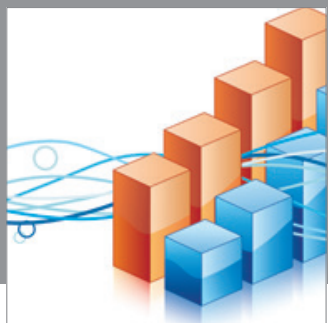

Advances in

Operations Research

mansans

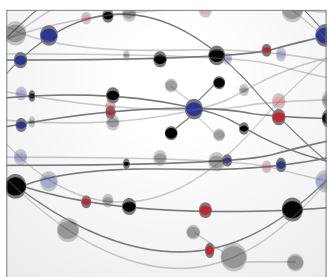

The Scientific World Journal
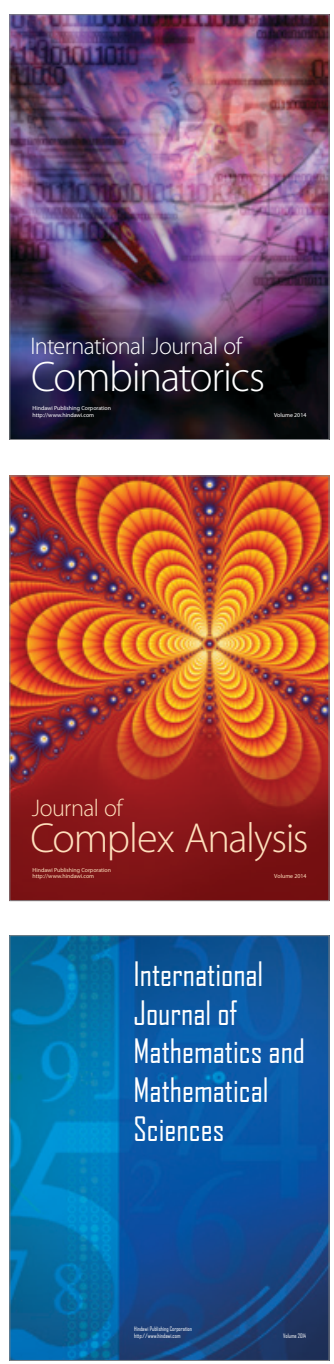
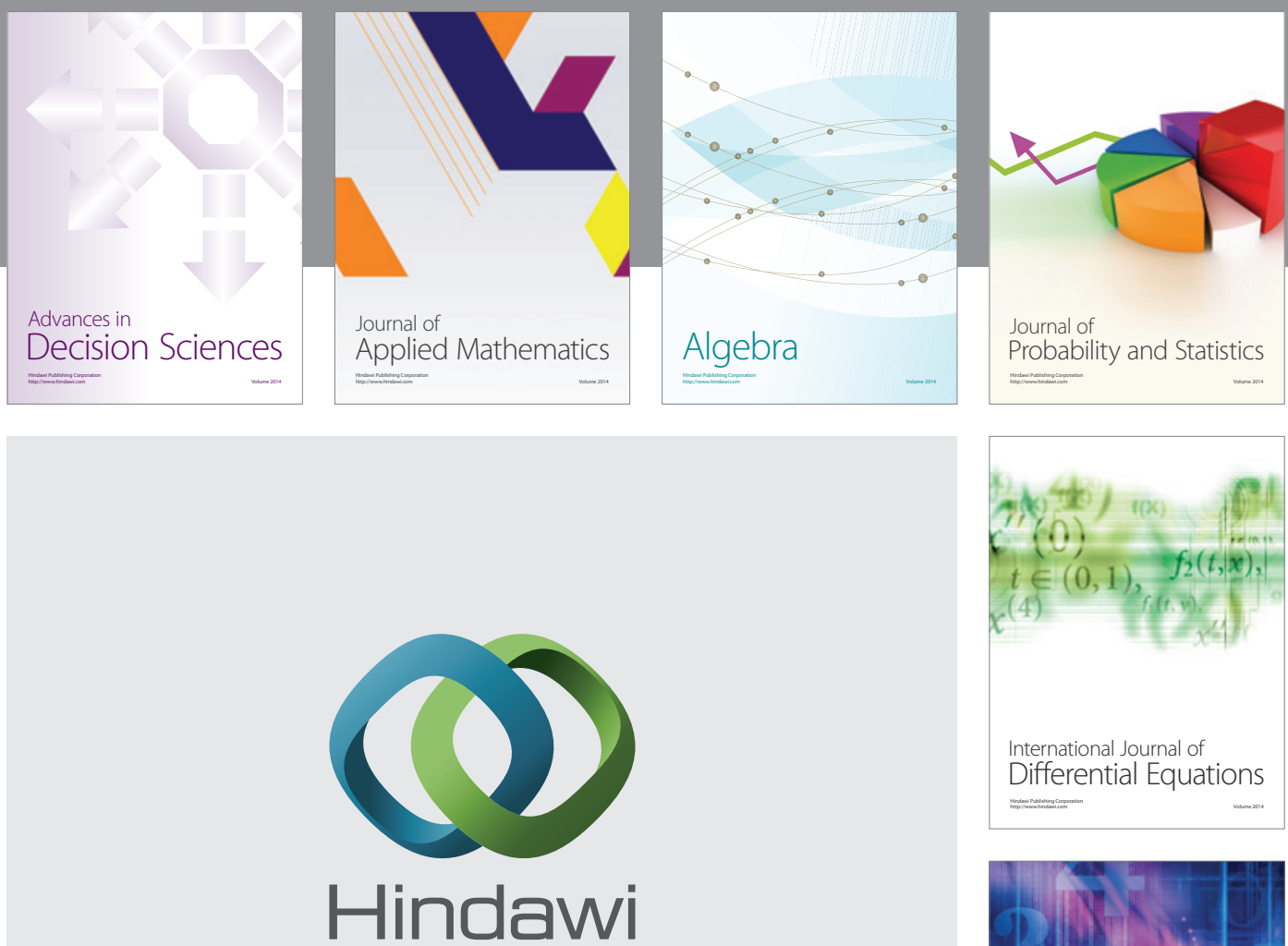

Submit your manuscripts at http://www.hindawi.com
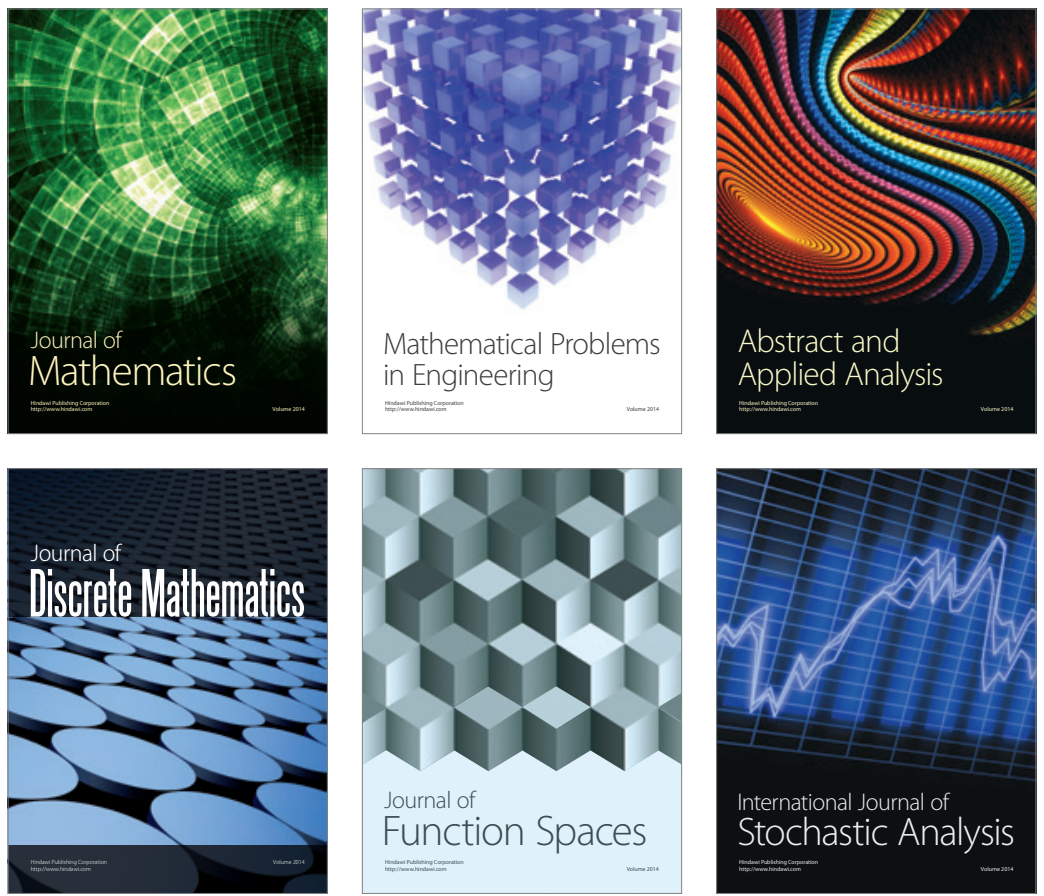

Journal of

Function Spaces

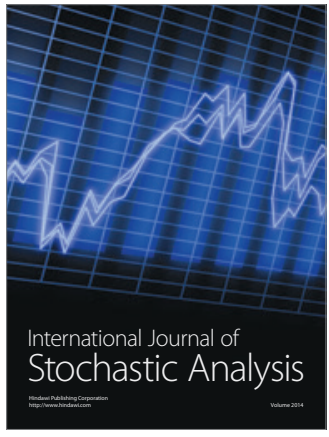

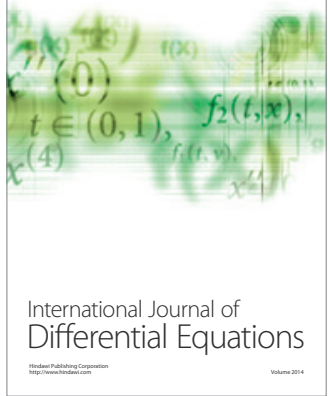
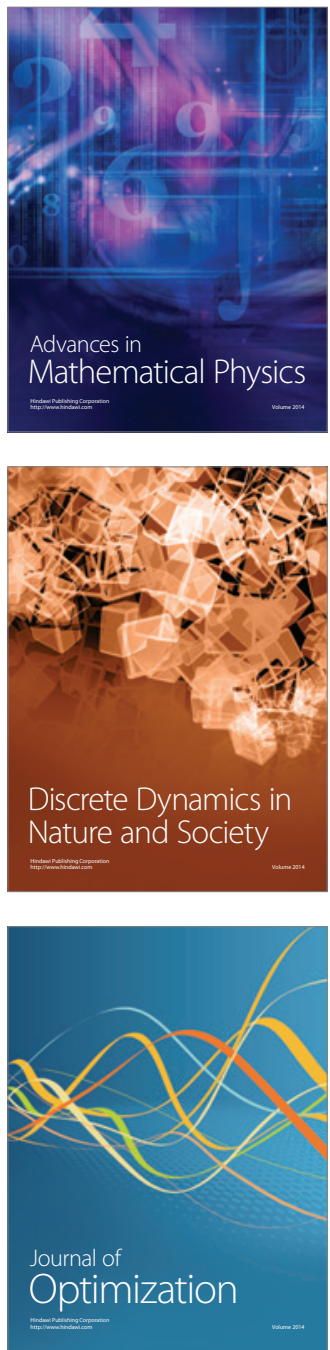